\title{
Impact of different confinement regimes on the two dimensional structure of edge turbulence
}

J A Alonso, S J Zweben ${ }^{1}$, P Carvalho ${ }^{2}$, J L de Pablos, E de la Cal, C Hidalgo, T Klinger ${ }^{3}$, B Ph van Milligen, $\mathbf{R} \mathbf{J}$

Maqueda $^{4}$, M A Pedrosa, C Silva ${ }^{3}$, M Spolaore ${ }^{5}, \mathbf{H}$

Thomsen $^{2}$ and the TJ-II team

Laboratorio Nacional de Fusión, Asoc. EURATOM-CIEMAT, Madrid, Spain

${ }^{1}$ Princeton Plasma Physics Laboratory, Princeton, New Jersey, USA

2 Ass. Euratom/IST, Centro de Fusão Nuclear, Instituto Superior Técnico,

Lisbon, Portugal

${ }^{3}$ Max-Planck IPP, EURATOM Ass., Greifswald, Germany

${ }^{4}$ Nova Photonics, Inc.,Princeton, New Jersey, USA

${ }^{5}$ Consorzio RFX, Associazione EURATOM-ENEA, Padova, Italy

E-mail: ja.alonso@ciemat.es 


\begin{abstract}
This paper reports the impact of different confinement regimes on the 2-D structure of edge turbulence. An image analysis method based on twodimensional continuous wavelet transformation (2D-CWT) is used to localize structures (blobs) in the images and to extract their geometrical characteristics (position, scale, orientation angle and aspect ratio). We study the impact of edge shear-layers on these geometrical aspects of blobs. Results show a reduction in the angular dispersion of blobs as the shear layer is established in the boundary, as well as an increase in the elongation of these structures. Similar behavior is found in NSTX image sequences when going from L to $\mathrm{H}$ mode plasmas. During improved confinement regimes the number of detected blobs decreases. Some indications are found suggesting that the turbulence reduction could be scale-selective in the biasing-induced improved confinement regime of TJ-II stellarator. Perpendicular flow reversal is visualized with the cameras and the time scales for flow reversal are found to be less than $50 \mu \mathrm{s}$. Radially propagating structures are found in the SOL with velocities in the range of $\sim 1000 \mathrm{~m} / \mathrm{s}$ and with a poloidally asymmetric spatial distribution.
\end{abstract}

\title{
1. Introduction
}

Transport in fusion devices is a phenomenon with high degree of complexity. Localized layers where $E \times B$ shear stabilization mechanisms are likely playing a role, have extensively been proved to have a beneficial impact in confinement. A reduction in turbulence amplitude is expected and measured [1]. However few attempts have been made to study the effects of such layers on the morphology of turbulent structures [2]. Two-dimensional images of edge plasma turbulence have been obtained by high-speed imaging in the visible range in the edge of tokamak devices [3], [4].

This paper reports a 2-D visualization of transport in the plasma edge of TJ-II stellarator. A wavelet-based image analysis method is used to localize and characterize blob-like structures. The impact of shear flows on turbulent structures is investigated by means of this method. Similar analyses are carried out in TJ-II and NSTX spherical tokamak and the results are compared.

\section{Experimental set-up}

Experiments were carried out in TJ-II plasmas Electron Cyclotron Resonance heated $\left(P_{E C R H}=200-400 \mathrm{~kW}, B_{T}=1 \mathrm{~T}, R=1.5 \mathrm{~m},\langle a\rangle=0.22 \mathrm{~m}, \frac{\iota(a)}{2 \pi} \approx 1.6\right)$. For the $2 \mathrm{D}$ turbulence studies presented here different cameras were used. Their names and characteristics are summarized in table 1. Cameras were placed on an optical bench one meter away from TJ-II stellarator central coil for them not to be affected by the prompt rise/fall of magnetic fields. The image was taken from the port to the camera sensor by means of a coherent fiber bundle and a suitable set of lenses.

Neutral recycling at the poloidal limiter is used to light up the outer plasma region $(\rho \sim 0.6-1)$. The view plane is a near-poloidal cross-section, the line of sight being approximately parallel to the local $B$ field direction (see Fig.1). The spread of the $\mathrm{H}_{\alpha}$ emission parallel to field lines around the poloidal limiter was also measured with another view to be about $10 \mathrm{~cm}$. The observed radial $\mathrm{H}_{\alpha}$ emission profile is almost flat in the first centimeters $(\sim 2-3 \mathrm{~cm})$ and then decaying with a typical decay length of about $5 \mathrm{~cm}$. 


\begin{tabular}{lccc}
\hline Name & Resolution @ Speed (exp. time) & Intensified & Frames/shot \\
\hline PSI-5 $^{(1)}$ & $64 \times 64 @ 250 \mathrm{kfps}(4 \mu \mathrm{s})$ & Yes & 300 \\
Photron APX-RS $^{(2)}$ & $80 \times 128 @ 100 \mathrm{kfps}(10 \mu \mathrm{s})$ & No & $\sim 10^{4}$ \\
Photron APX-I ${ }^{(2)}$ & $80 \times 256 @ 50 \mathrm{kfps}(1 \mu \mathrm{s})$ & Yes & $\sim 10^{4}$ \\
\hline
\end{tabular}

(1) Princeton Scientific Instruments

Table 1. Fast camera models used for the results presented in this Paper.

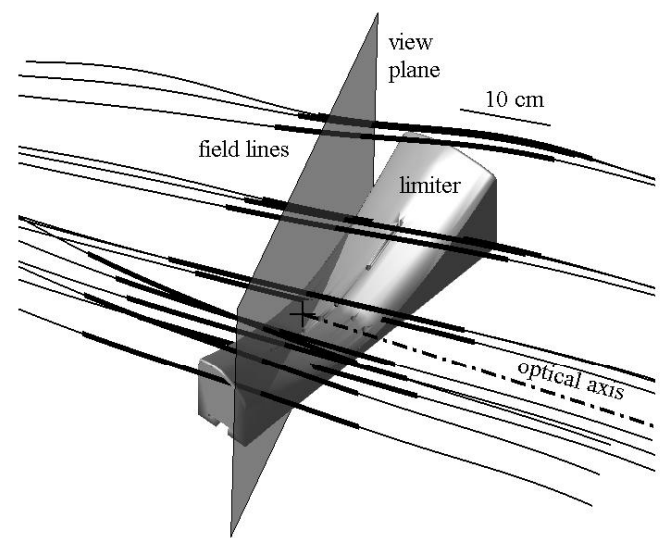

Figure 1. View plane and field lines by the poloidal limiter used for plasma edge lightening. Broad lines stand for the toroidal extent of the light cloud.

Bright structures are frequently seen with a spatial extent of a few centimeters. These structures show predominant poloidal movements with typical speeds of $10^{3}$ $10^{4} \mathrm{~m} / \mathrm{s}$ in agreement with the expected $\mathbf{E} \times \mathbf{B}$ drift rotation direction.

\section{Blob geometry}

\subsection{Geometrical parameters and feature extraction method}

The ellipse-like structures seen in the background-subtracted $\ddagger$ images motivate the definition of the following geometrical parameters: a) scale or size of the blob b) orientation angle (angle with respect to horizontal axis of the ellipse's principal axis) c) aspect ratio (ratio of the principal axis scale to the scale in the direction perpendicular to this).

A two-dimensional continuous wavelet transformation (2D-CWT) was found to be suitable for extracting these parameters. A thorough introduction to the 2D-CWT can be found in [5]. We outline here only the necessary formalism to make the following explanation clear. The transformation is given by:

$$
S(a, \vec{b})=\left\langle\psi_{a, \vec{b}}(\vec{x}) \mid f(\vec{x})\right\rangle,
$$

where $\psi(\vec{x})$ is the analysing wavelet and $\psi_{a, \vec{b}}(\vec{x})=\frac{1}{a} \psi\left(\frac{\vec{x}-\vec{b}}{a}\right)$, whereas $f(\vec{x})$ is the image function. Angle brackets stand for spatial integration. In the two dimensional case, anisotropic wavelets can be constructed, the transformation being labeled with an additional angle parameter $\theta, \psi_{a, \vec{b}, \theta}(\vec{x})=\frac{1}{a} \psi\left(\frac{r_{-\theta}(\vec{x}-\vec{b})}{a}\right)$, where $r_{-\theta}$ is the $\theta$-rotation matrix. This allows the extraction of oriented features.

$\ddagger$ Background is calculated as the median of every pixel's time trace in a $1 \mathrm{~ms}$ time window. 
The method presented here has a detection-recognition scheme based on isotropic (detection) and anisotropic (recognition) 2D-CWT [6]. In a first stage blobs are localised. For this purpose, the image is wavelet-transformed with a Mexican hat wavelet,

$$
\psi^{\text {MexHat }}(x, y)=C\left(2-x^{2}-y^{2}\right) e^{-\frac{x^{2}+y^{2}}{2}},
$$

where $C$ is a normalizing factor. The result contains original image structures with a scale comparable to that of the analysing wavelet. Weak structures are removed after thresholding. The threshold is chosen higher for smaller scales which are more affected by noise. Local extrema are regarded as blob positions $\vec{b}_{j}, j=1 . . N_{\text {blobs }}$. The characteristics of the Mexican Hat fourier spectrum together with the frame size and the pixel resolution limit the number of scales to be used. For the results presented here three scales separated by a factor of two were selected in the range between 0.7 $\mathrm{cm}^{-1}$ to $2.8 \mathrm{~cm}^{-1}$.

At a second stage the Morlet wavelet,

$$
\psi^{\text {Morlet }}(x, y)=C\left(\cos \left(k_{0} y\right)-e^{-\frac{1}{2} k_{0}^{2}}\right) e^{-\frac{1}{2}\left(\epsilon^{-1} x^{2}+y^{2}\right)},
$$

was used for directional analysis. The parameters $k_{0}$ and $\epsilon$ were set to 3 and 1 respectively. This choice was a compromise between angular resolving power and spatial locality. Angular convolution with the Morlet wavelet gives the angular response curve of the $j$-th blob detected $W^{(j)}(\theta)=\left\langle\psi_{a, \vec{b}_{j}, \theta}^{\text {Morlet }}(\vec{x}) \mid f(\vec{x})\right\rangle$. From this curve, a blob's orientation angle $\hat{\theta}$ can be inferred. $\hat{\theta}$ is the angle between blob's principal axis and the frame x-axis. No precise mapping of magnetic surfaces over the images was yet made, so that scales and angles presented here are not referred to local magnetic coordinates (i.e. radial-poloidal coordinates) but to frame coordinates.

The aspect ratio (the ratio of blob dimension along its principal axis to the dimension along an axis perpendicular to this) can be estimated as $A R^{(j)}=$ $\sqrt{\frac{W^{(j)}(\hat{\theta})}{W^{(j)}(\hat{\theta}+\pi / 2)}}$.

\subsection{Edge shear layer effect on blob geometry}

The appearance of a natural shear layer in the TJ-II stellarator above a density threshold (though probably density is only an operational control parameter) is a robust and well characterised phenomenon [7]. The threshold density is found around $0.6 \times 10^{13} \mathrm{~cm}^{-3}$ for the magnetic configuration used for the experiments reported in this paper. Bellow this density, the Langmuir and Heavy Ion Beam probes measure the positive central electric potential to monotonically decay towards the edge, smoothly continuing the flat potential profile in the SOL. For densities higher than the threshold density the radial electric field becomes negative (radially inwards) in the boundary and the shear in the $E \times B$ velocity profile increases in the edge region $(r / a \sim 0.9)$.

Fast imaging of the plasma edge in different shots with distinct densities revealed an effect of the shear layer on turbulent structures. Results are summarized in figure 2 .

The angular distribution referred to in figure 2.a is the distribution of the orientation angles (as defined in section 3.1) of all the detected blobs with an aspect ratio above 2 . The standard deviation of this angular distribution is a measure of the scatter in the orientation, i.e. the lower the standard deviation the more uniformly 

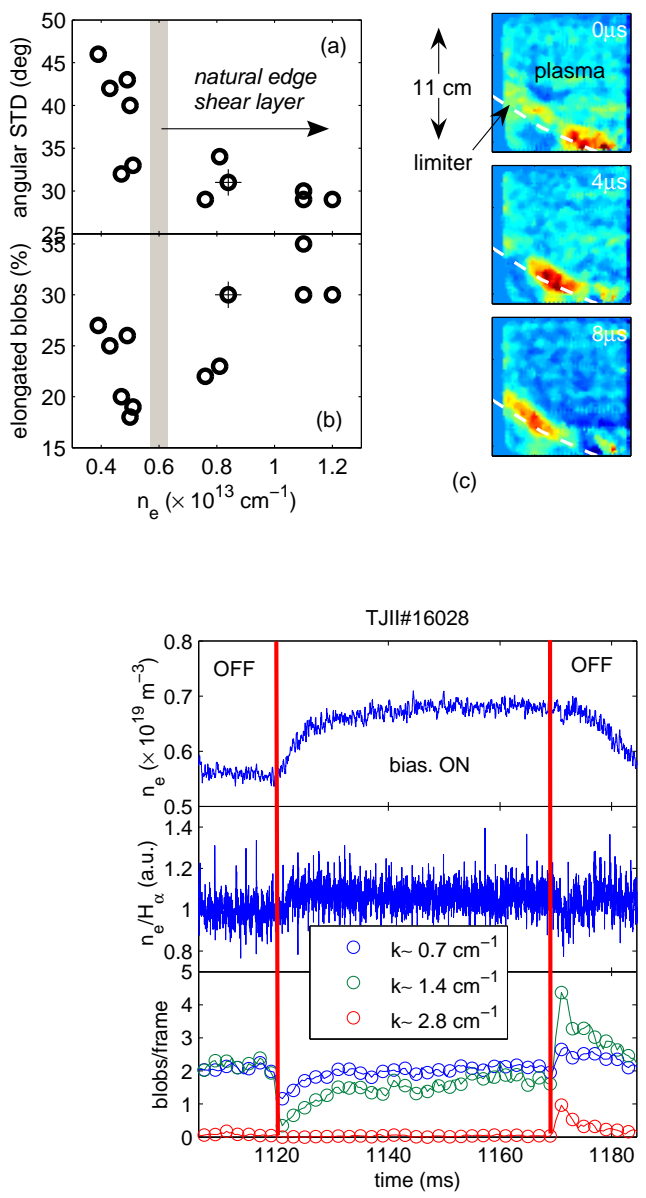

Figure 2. Effect of edge shear layer on blob geometry. The standard deviation of the distribution of blobs' angles decreases for increasing density (a). The percentage of elongated blobs has a slight increasing tendency for increasing density (b). (c) Sample image series for the shot 12369 marked with a plus sign in plots (a),(b) Camera: PSI5 (see table 1). Grey stripe stands for the threshold density for shear layer development. The scale of the structures used for statistics was $k=1.2 \pm 0.5 \mathrm{~cm}^{-1}$

\section{Figure 3.}

Evolution of line averaged electron density, electron density to $\mathrm{H}$ alpha ratio and blob population during a biasing experiment (TJ-II shot numebr 16028, electrode $2 \mathrm{~cm}$ inside the plasma, biasing voltage $300 \mathrm{~V})$. Camera: Photron APX-RS, 8064 frames@100 kfps $(10 \quad \mu$ s exp. time).

aligned the blobs are. The fraction of such elongated blobs $(A R>2)$ is the 'percentage of elongated blobs' and is a measure of blobs' elongation degree. According to this interpretation, the effect of the shear layer is to align the orientation of the blobs as well as to stretch them.

\subsection{External biasing induced improved confinement regime}

Improved confinement regimes are accessible in TJ-II stellarator through external biasing [8]. Plasma edge is biased with a graphite electrode installed in a fast reciprocating probe drive. The electrode was inserted typically $2 \mathrm{~cm}$ inside the LCFS and biased with respect to one of the two TJ-II limiters [9]. Significant modifications in the level of fluctuations have been previously reported using probe measurements during the transition to improved confinement regimes induced by edge biasing $[8,9]$.

Figure 3 shows the time evolution of line averaged plasma density $\left(n_{e}\right)$, the ratio $n_{e} / H_{\alpha}$ and the blob populations detected in three different scales during edge biasing. During the biasing phase, image fluctuations decrease in intensity and fewer events are detected by the extraction method. 
There are some indications that the reduction in turbulence levels is not the same for all the scales. From the evolution of blob population of figures 3 it can be noticed that the reduction (or increase in the case of biasing turn-off) for medium scale structures is somewhat stronger than for big scale ones. The $k$ power spectra of the on/off biasing phases exhibits this effect only marginally. There exists a relatively higher reduction/increase around $k \sim 1.5 \mathrm{~cm}^{-1}$ but this difference is within the error bars.

\subsection{Blob statistics during $L$ and $H$ modes in NSTX}

The blob extraction / analysis method presented in section 3.1 was applied to images of the edge of the NSTX spherical tokamak obtained with active gas puffing technique [3]. Recordings of $\mathrm{L}$ mode plasmas show an intense blob activity in the edge of the plasma with structures coming out to the SOL. H mode images show little blob activity in the edge with rare expulsion of structures from the boundary. Figure 4.d shows one of these structures in an L mode sequence. Figure 4.e shows a few frames from a $\mathrm{H}$ mode shot.

Differences were found in the geometric properties of the blobs detected in $\mathrm{L}$ and $\mathrm{H}$ mode plasmas as represented in figures 4.a, b. The standard deviation of the blobs' angular distribution is significantly smaller for $\mathrm{H}$ mode plasmas whereas the percentage of elongated blobs increases from $\mathrm{L}$ to $\mathrm{H}$ mode pulses. These effects are qualitatively similar to those found with the establishment of the natural shear layer in TJ-II (section 3.2) though quantitatively stronger.

Figure 4.c is the number of the events per frame where the above-mentioned reduction of turbulence activity is made evident.

\subsection{Comment on analysis and results}

Fast moving blobs can appear in the images as blurred elongated structures. The poloidal velocities measured in the TJ-II stellarator with Langmuir probes are in the range of $1000 \mathrm{~m} / \mathrm{s}$ for low density regimes and $-1000 \mathrm{~m} / \mathrm{s}$ for high density regimes. Typical values for poloidal rotation during the external biasing improved confinement regime are about $1500 \mathrm{~m} / \mathrm{s}$. In the case of NSTX, poloidal velocities as measured with a poloidal array of $\mathrm{H}_{\alpha}$ chords show a big dispersion for $\mathrm{L}$ mode plasmas but tend to cluster around $-4000--3000 \mathrm{~m} / \mathrm{s}$. For $\mathrm{H}$ mode plasmas, the measured velocities are in the range of $-4000--5000 \mathrm{~m} / \mathrm{s}$ (see [3]). Therefore the poloidal velocities of the regimes compared in this paper are rather similar and thus the blurring effect should be equally affecting to the measurements. Even a $1000 \mathrm{~m} / \mathrm{s}$ difference in poloidal velocities would cause a $1 \mathrm{~cm}$ movement during $4 \mu$ s integration time. For a blob scale of $\lambda \sim 5 \mathrm{~cm}\left(k \sim 1.2 \mathrm{~cm}^{-1}\right)$ and $A R=1$ would be seen as a blob with $A R=1.08$. This effect decreases for increasing $A R$ and cannot account for the observed differences in the aspect ratio and orientation angle histograms.

The recordings analysed here were taken in different density and confinement regimes. In spite of this variety of plasma conditions the radial extent of the light cloud (i.e. the region where the $\mathrm{H}_{\alpha}$ signal/noise ratio is high enough for the structures to be detected) did not change considerably. 


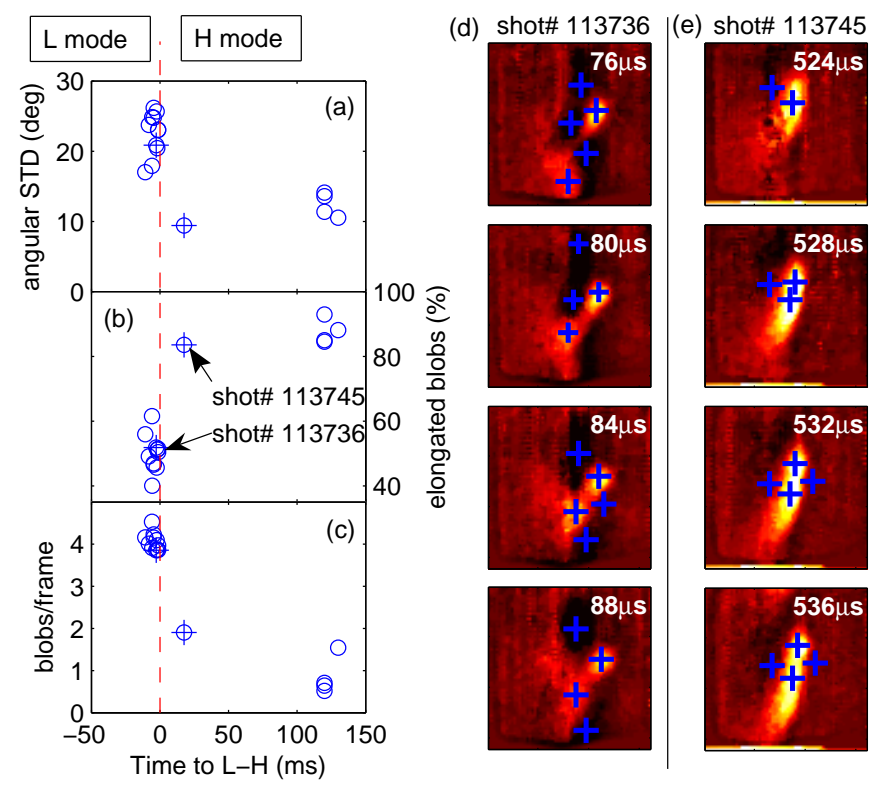

Figure 4. $k=(1.2 \pm 0.5) \mathrm{cm}^{-1}$ blobs statistics for different NSTX shots in L and $\mathrm{H}$ mode regimes (a)-(c). Image series (e) and (d) are sample sequences for the shots marked in (b). Detected $k \sim 1.2 \mathrm{~cm}^{-1}$ structures are marked with a blue cross. The frame side length is $23 \mathrm{~cm}$. Camera: PSI-5 (see table 1).

\section{Time scales}

Previous works have reported on the existence of two different time scales in the evolution of plasma edge parameters (e.g. floating potential) during the formation of the sheared flow pattern [10]. The change in the floating potential during the density ramp-up is completed in a few tens of milliseconds. Together with this slow reversal, fast transients (in the scale of few tens of microseconds) are observed in the proximity of the threshold density.

These fast transients have now been assessed with fast camera images. For densities below the above-mentioned threshold, the structures rotate counter-clockwise in the poloidal direction (figure 5.a) in the direction of the $E \times B$ velocity. When the density is close to the threshold the rotation direction becomes uncertain and either of the two rotation directions can be distinguished for a few frames. Furthermore, a rotation inversion can be captured as figure 5.b illustrates. This inversion takes place within a few tens of microseconds and thus supports previous observations of fast transients in probe signals.

For higher densities, electric fields in the boundary become negative and the structures rotate clockwise (figure 5.c).

\section{Radial transport and poloidal asymmetries}

The poloidal limiter proved to be a good source of neutrals for imaging of $\mathrm{H}_{\alpha}$ in the outer part of the plasma. However, in order to image the SOL it was necessary to 


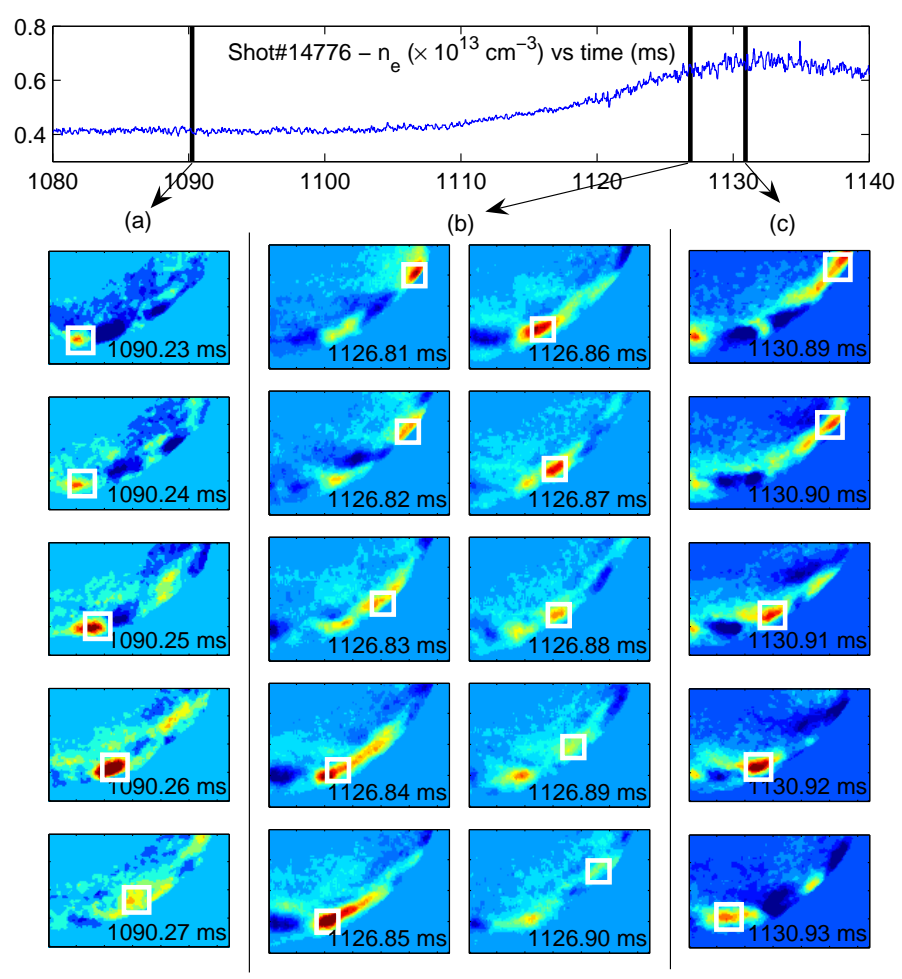

Figure 5. Evolution of poloidal movement of structures in different time segments during a density ramp-up. (a) Positive electric field, (b) Transient phase where poloidal velocity jumps from positive to negative in a few tens of microseconds. (c) Regime with fully developed negative electric field. Camera: Photron APX-RS (see table 1).
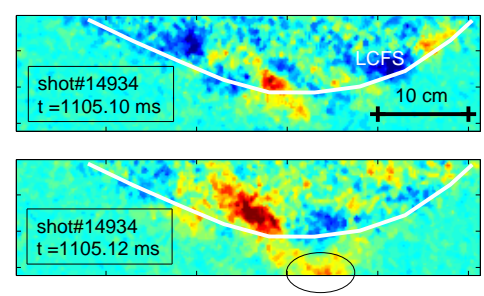

Figure 6. Two consecutive frames for estimating the radial velocity of blobs. Structure within the ellipse is absent $20 \mu \mathrm{s}$ before. Camera: Photron APX-I (see table 1).

increase the plasma-limiter separation. The reduced signal level made it necessary to use an intensified camera.

With this experimental set-up structures are found in the SOL (see figure 6). The spatial distribution of the observed structures in the SOL is not poloidally symmetric. Detected events seem to cluster in the lower region of the frame where the magnetic field is lower. Nevertheless, a systematic study of the relationship between the poloidal distribution of structures and magnetic topology remains to be done.

From figure 6 , an estimation for the radial velocity of one blob can be calculated. The structure marked with an ellipse in the lower image is several centimeters away 
from any other structure in the previous frame (figure 6 upper image). Then, assuming that this structure came radially out of the plasma column, $v_{r} \approx 2 \mathrm{~cm} / 20 \mu \mathrm{s}=1000 \mathrm{~m} / \mathrm{s}$. This coarse estimation is in the tail of the perpendicular velocities probability distribution functions calculated from the perpendicular particle flux induced by fluctuations $\left(v_{r}=\left\langle\Gamma_{E \times B}\right\rangle /\langle n\rangle\right)$.

The dependence of the radial velocity on the structure's scale is of importance not only for understanding the transport in the SOL but also to predict particle and energy losses onto divertor plates and main chamber wall in fusion devices. From the theoretical point of view, some models conclude that the radial speed increases as the square root of the blob size [11] whereas others [12] predict a radial blob velocity inversely proportional to the blob size squared. Fast imaging diagnostics together with multi scale techniques, as the one outlined in section 3.1, can serve to unravel the dispersion relation $v(k)$ of the SOL propagating structures.

\section{Conclusions}

Two dimensional plasma edge turbulence was investigated by means of fast imaging in the visible range. Both radial and poloidal dynamics have been investigated. Observed structures velocities are $v_{r} \sim 1 \mathrm{~km} / \mathrm{s}$ and $v_{\theta} \sim 1-5 \mathrm{~km} / \mathrm{s}$.

A continuous wavelet-based method was used to localize and study the geometry of spatially coherent turbulent structures (scale, aspect ratio and orientation angle) in different plasma regimes.

The impact of the naturally occurring shear-layer, self-organized near marginal stability, on TJ-II edge turbulent structures was addressed. Experimental results show a reduction in the angular dispersion of $k \sim 1.2 \mathrm{~cm}^{-1}$ blobs as the shear layer is established in the boundary, as well as a slight though noticeable shift of the aspect ratio histogram toward higher values. A similar effect is observed in NSTX image data when going from $\mathrm{L}$ to $\mathrm{H}$ mode plasmas. These results are consistent with the picture of the shear layer stretching blobs as well as ordering them.

Secondly the effect of external biasing on the relative populations of blobs of three different scales was studied. A reduction in all the analysed scales is observed during the biasing phase. Some indications are found suggesting that the turbulence reduction could be scale-selective.

The plasma poloidal flow inversion when going through the TJ-II critical density for shear layer formation was captured. The flow reversal was seen to take a few turbulence autocorrelation times $(\sim 50 \mu \mathrm{s})$.

In order to study the SOL structures it is necessary to operate with intensified cameras and/or active gas puffing injection. Structures seen in the TJ-II scrape-off layer have speeds about $1 \mathrm{~km} / \mathrm{s}$ and their spatial distribution is poloidally asymmetric.

\section{References}

[1] Terry P W 2000 Rev. Mod. Phys. 72(1) 109-165

[2] Thomsen H 2005 in Proc. 32nd EPS conference on Control. Fusion and Plasma Physics (Tarragona: ECA) p P5.027

[3] Zweben S J et al 2004 Nucl. Fusion 44 134-153

[4] Terry J L et al 2003 Phys. Plasmas 10(5) 1739-1747

[5] van den Berg J C, ed 1999 Wavelets in Physics (Cambridge CB2 2RU, UK: Cambridge University Press)

[6] Antoine J P 1998 CWI Quarterly 11(4) 323-345 
[7] Pedrosa M A et al 2005 Plasma Phys. Control. Fusion 47 777-788

[8] Hidalgo C et al 2004 Plasma Phys. Control. Fusion 46 287-297

[9] Silva C et al 2005 in Proc. 32nd EPS conference on Control. Fusion and Plasma Physics (Tarragona: ECA) p P2.037

10] Gonçalves B et al 2006 Phys. Rev. Lett. 96145001

[11] Garcia O E et al 2005 Phys. Plasmas 12090701

[12] Krasheninnikov S I 2001 Physics Letters A 283368 